\title{
Perfiles Motivacionales del Profesorado ante la Formación en Metodologías Activas en la Universidad
}

\author{
Clemente Lobato ${ }^{(1) *}$ y Alba Madinabeitia ${ }^{(2)}$ \\ (1) Facultad de Filosofía y CC de la Educación, Dpto. de Psicología Evolutiva y de la Educación, \\ Universidad del País Vasco-Euskal Herriko Unibertsitatea, Av. De Tolosa, 70, 20018, \\ Donosita, San Sebastián-España (e-mail: clemente.lobato@ehu.es) \\ (2) Servicio de Asesoramiento Educativo, Universidad del País Vasco-Euskal Herriko \\ Unibertsitatea, Edificio de la Biblioteca, 5, 48940, Leioa-España. \\ (e-mail: alba.medinabeitia@ehu.es) \\ * autor a quien debe ser dirigida la correspondencia
}

Recibido Ene. 10, 2011; Aceptado Ene. 30, 2011; Versión final recibida Feb. 16, 2011

\begin{abstract}
Resumen
Se presenta un estudio para determinar que motivaciones llevan a comprometerse a los docentes universitarios con este proceso de formación de competencias. En el contexto actual la Universidad del País Vasco en España ha decidido capacitar a su profesorado ante el reto que supone la formación de competencias a través del trabajo y aprendizaje autónomo del estudiante por medio del programa Eragin de metodologías activas. En este desarrollo profesional la motivación docente cobra una importancia singular, asunto que es discutido en este trabajo. La conclusión principal sostiene que los docentes participantes en este proceso formativo de cambio metodológico están intrínsecamente muy motivados. Y a pesar de que en este proceso de formación las motivaciones extrínsecas están presentes, éstas quedan relegadas a un segundo plano.
\end{abstract}

\section{Motivational Profiles of the Teaching staff towards the Training on Active Methodologies within the University}

\begin{abstract}
A study is presented to analyze and determine which kind of motivation leads the teaching staff to get actively involved in the process of teaching of competences. Presently, the Basque Country University in Spain has decided to provide its teaching staff with a competence-based training through the Eragin Program of active methodology oriented towards the self-learning of the student. In this professional development, teaching motivation becomes of particular importance, subject that is discussed in this work. The main conclusion is that the professors involved in this training process of methodological change are intrinsically motivated. And even though the extrinsic motivations are present in this training process, they do not play an important role.
\end{abstract}

Keywords: teaching motivation, active teaching methodology, competences, professional development 


\section{INTRODUCCIÓN}

Actualmente hemos de enfrentarnos a nuevos retos ante los numerosos y diversos cambios surgidos en la sociedad. Y la educación tiene el cometido de educar a los sujetos en esas nuevas claves de la sociedad del conocimiento. En la comunidad universitaria se está dando una profunda reflexión y un cambio de mentalidad planteando algunos interrogantes: ¿Qué significa enseñar en la sociedad del conocimiento?, ¿y cómo se aprenden de forma autónoma las competencias profesionales?, ¿cuál es el rol del profesor y del alumno hoy en día en la Universidad?

Encarando este reto, la Universidad del País Vasco/EuskalHerriko Unibertsitatea (UPV/EHU) ha elaborado su propio modelo de educación, 'ikd' - aprendizaje cooperativo y dinámico-, en cuyo desarrollo han de converger cuatro factores: desarrollo institucional, desarrollo social y territorial, educación activa y desarrollo profesional. A este último pertenece el programa de formación en metodologías activas de enseñanza ERAGIN, que contribuye a la línea estratégica de estimular y apoyar el aprendizaje dinámico y cooperativo de los estudiantes por medio de la formación del profesorado, eslabón fundamental de este cambio para una mayor calidad en la educación. El desarrollo profesional de los docentes (Uwamariya y Mukamurera, 2005), se refiere al crecimiento profesional que logra un profesor como consecuencia del análisis sistemático de su experiencia y práctica, en las que emergen factores externos e internos, entre otros la motivación (Ollarves, 2006). De ahí, la importancia de investigar el perfil motivacional de los docentes universitarios, ya que constituye una variable predictiva de la forma de trabajar del docente (Silvero, 2006).

El objetivo de esta investigación pretende conocer las motivaciones iniciales del profesorado participante en el programa de formación ERAGIN impulsado por la UPV/EHU. En primer lugar, revisamos las teorías y conceptos fundamentales de la motivación y analizamos algunas investigaciones sobre las motivaciones del profesorado ante su formación permanente. En segundo lugar, exponemos el desarrollo empírico con la formulación de las conclusiones del estudio.

\section{Motivación para el trabajo}

La psicología ha abordado la motivación desde perspectivas diversas partiendo del modelo sistémico (Barberá y Mateos, 2000). Es un constructo teórico, hipotético y no observable (Muchinsky, 2000), en el que el acercamiento conceptual y empírico no ha sido uniforme (De la Fuente, 2004). Diversos autores (De la Fuente, 2004; Añez, 2006; González Fernández, 2007; Lozano y Repetto, 2007) comparten una descripción que podemos formular en estos términos: la motivación en el trabajo es un conjunto de fuerzas muy eficientes que se originan dentro y más allá del ser individual para iniciar la conducta relacionada con el trabajo y para determinar su forma, dirección, intensidad y duración.

En una de las escasas investigaciones en torno a nuestro objeto de estudio, González Torres (2003) sostiene que las personas desarrollan tres tipos de relación con su trabajo: como trabajo, como carrera o como vocación, que predicen las metas que la gente persigue en el mismo. Quienes experimentan su profesión como trabajo se centran en los aspectos económicos más que en el placer o realización personal. Los que lo ven como carrera, en el avance profesional dentro de la estructura ocupacional. Y por último, quienes lo definen como vocación, en el disfrute que acarrea su realización y en el trabajo socialmente útil. Según varios autores (Bastick, 2000; Biscarri et al., 2006; Kenneth, 2002), la motivación docente está basada principalmente en elementos intrínsecos: el reto intelectual, la autonomía, la libertad para probar nuevas ideas, el desarrollo de la competencia profesional y la oportunidad de crecer personalmente. En este sentido existen investigaciones (Antón, 2005; Bastick, 2000; Biscarri et al., 2006; Añez, 2006 y Sinclair, 2006) que profundizan las motivaciones intrínsecas y extrínsecas del profesorado.

\section{Motivaciones docentes para la formación docente}

La motivación del profesorado para enseñar ha recibido escasa atención (Vezub, 2009). Y menos aún las motivaciones respecto a su formación permanente. En las investigaciones realizadas, preferentemente desde perspectivas cuantitativas con el análisis factorial, con profesorado de 
enseñanza secundaria y universitaria ante su formación permanente, se ha tratado de identificar y clasificar diversos tipos de motivaciones (Manuel y Hughes, 2006).

Algunos trabajos (Biscarri, 1993; García, 1998; Ryan, 2003; Biscarri et al., 2006; Manuel y Hughes, 2006; Bruinsma y Jansen, 2010) comprueban que las motivaciones intrínsecas se sitúan por encima de las extrínsecas. De hecho, una de las motivaciones más sobresalientes es la actualización del conocimiento científico y las destrezas profesionales. Los incentivos relacionados con la promoción del estatus profesional quedan relegados a un segundo plano. Sin embargo, otras investigaciones (Vezub, 2009) concluyen que la razón de no pocos docentes para participar en programas de formación responde a un interés de promoción profesional a través de un sistema de puntos, incentivos o incluso la opción de acceder a mejores puestos de trabajo. De todos modos, no hay duda, según Ryan, (2003) y Burinsma y Jansen, (2010), de que la motivación para comprometerse con un proceso de formación encaminado al desarrollo profesional, puede ser intrínseca y extrínseca a la vez. Biscarri (1993) confirma que tanto una como otra juegan un papel importante en las valoraciones subjetivas de los docentes ante actividades de formación.

Los estudios realizados para conocer la motivación de los docentes universitarios frente a su formación permanente, son aún más escasos (Silvero, 2006). No obstante, Anton (2005) deja claro que desde 2003 la formación continuada del profesorado cobra mayor relevancia y se convierte en un elemento imprescindible para la mejora de la calidad de la educación universitaria. Como insisten Vezub (2009) y Bruinsma y Jansen (2010) la actualización del cuerpo docente y la promoción del debate pedagógico son necesarias si queremos mejorar la calidad de la educación universitaria. Ante este panorama, parece necesario abordar el tema de la motivación ligada al ejercicio docente, y más concretamente, a la motivación centrada a la formación permanente. Por eso nos preguntamos: ¿qué razones impulsan al profesorado de la UPV/EHU a comprometerse con el proceso de formación ERAGIN?, ¿cuáles son los perfiles motivacionales que manifiestan? Entendemos que estas cuestiones están estrechamente vinculadas al desarrollo profesional docente (Biscarri, 1993).

\section{METODOLOGÍA}

Abordamos el estudio desde una perspectiva descriptiva para lograr el objetivo general de determinar la motivación que el profesorado declara tener al iniciar un proceso formativo en el ámbito de la innovación docente. Para alcanzar este objetivo general se proponen los siguientes objetivos específicos: a) Identificar las motivaciones iniciales del profesorado universitario ante un proceso de formación en innovación docente; b) Analizar el tipo de motivación según variables como el sexo, la edad, los años de experiencia docente universitaria y el área de conocimiento; c) Describir el perfil motivacional del profesorado universitario que apuesta por las metodologías activas de enseñanza.

Muestra: El número de participantes en esta primera promoción del Programa ERAGIN es de 75. Se han seleccionado teniendo en cuenta estos criterios: i) Representación de titulaciones y áreas de conocimiento presentes en la UPV-EHU; ii) Experiencia previa como formadores de profesores y en innovación docente; y iii) Priorización de profesores con docencia en primer curso de una titulación.

Instrumentos: Para este estudio elaboramos el cuestionario MOTIGU. En una primera fase se revisó y analizó la motivación docente hacia la formación profesional (Bastick, 2000; González, 2003; Antón, 2005; Biscarri et al., 2006; Rosales, 2006, Bruinsma y Jansen, 2006) estableciéndose tres dimensiones motivacionales: Carencias competenciales, Promoción profesional y Desarrollo personal, con las que se formularon 14 ítems de respuesta tipo (Likert 15) y una pregunta abierta. Se recogieron algunos datos identificativos (sexo, antigüedad docente, área de conocimiento...). En un segundo momento, el cuestionario fue revisado por dos expertas docentes e investigadoras universitarias que introdujeron algunas modificaciones y posteriormente aprobaron la versión definitiva. 
Procedimiento: La recogida de información se ha llevado a cabo el primer día del programa de formación antes de comenzar la primera sesión. Los datos obtenidos en los ítems se han tratado con el programa estadístico SPSS versión 17. En el caso de la cuestión abierta, la información se ha analizado con el programa informático para el análisis cualitativo Nvivo.

\section{RESULTADOS. ANÁLISIS Y DISCUSIÓN}

En primer lugar se analizan los resultados obtenidos de los primeros estadísticos que se muestran en la Tabla 1. En un primer lugar se aprecia una cierta heterogeneidad en las valoraciones obtenidas, oscilando las puntuaciones medias alcanzadas por cada ítem entre 2,51 y 4,75. En las desviaciones típicas, también existe cierta diferencia: la dispersión se encuadra entre 0,47 y 1,15. Ambos intervalos son indicativos de la existencia de cierta diferencia entre los datos.

Tabla 1: Resultados. Primeros estadísticos

\begin{tabular}{|l|c|c|c|c|c|}
\hline & $\mathrm{N}$ & Mín & Máx & $\mathrm{M}$ & D. T. \\
\hline $\begin{array}{l}\text { MOTIV02 Me he apuntado a este proceso para cubrir mis carencias } \\
\text { competenciales (falta recursos pedagógicos, habilidades docentes...) }\end{array}$ & 71 & 1 & 5 & 3,61 & 1,15 \\
\hline $\begin{array}{l}\text { MOTIV03 Me he involucrado en este proceso formativo para facilitar } \\
\text { mi promoción profesional, mejorar mi currículum, los incentivos... }\end{array}$ & 70 & 1 & 5 & 2,51 & 1,15 \\
\hline $\begin{array}{l}\text { MOTIV04 Mi preocupación principal es desarrollarme como persona } \\
\text { (crecimiento, compromiso, responsabilidad personal...) }\end{array}$ & 71 & 2 & 5 & 3,70 &, 99 \\
\hline $\begin{array}{l}\text { MOTIV05 Las características de mi labor docente en la universidad } \\
\text { (trabajar con estudiantes, dar clase)me motivan por sí mismas }\end{array}$ & 71 & 2 & 5 & 4,46 &, 60 \\
\hline $\begin{array}{l}\text { MOTIV06 Me motiva el status del profesor universitario } \\
\text { (reconocimiento social...) }\end{array}$ & 71 & 1 & 5 & 2,54 & 1,04 \\
\hline $\begin{array}{l}\text { MOTIV07 Me siento empujado/a institucionalmente a participar en } \\
\text { este tipo de iniciativas }\end{array}$ & 70 & 1 & 5 & 2,61 & 1,14 \\
\hline $\begin{array}{l}\text { MOTIV08 Considero que la formación es necesaria para ser docente } \\
\text { competente }\end{array}$ & 71 & 2 & 5 & 4,58 &, 66 \\
\hline $\begin{array}{l}\text { MOTIV09 Me preocupa que el alumnado logre un aprendizaje y } \\
\text { desarrollo de las competencias personales y profesionales }\end{array}$ & 71 & 3 & 5 & 4,75 &, 47 \\
\hline $\begin{array}{l}\text { MOTIV10 Me interesa dinamizar grupos de docentes preocupados } \\
\text { por el cambio metodológico }\end{array}$ & 71 & 2 & 5 & 4,03 &, 91 \\
\hline $\begin{array}{l}\text { MOTIV11 Propuestas como ERAGIN me resultan motivadoras para } \\
\text { mi desarrollo profesional }\end{array}$ & 71 & 3 & 5 & 4,18 &, 68 \\
\hline $\begin{array}{l}\text { MOTIV12 Participo en esta formación para NO quedarme al margen } \\
\text { de los cambios }\end{array}$ & 69 & 1 & 5 & 3,42 &, 96 \\
\hline $\begin{array}{l}\text { MOTIV13 La formación docente aumenta mi prestigio como profesor } \\
\text { universitario }\end{array}$ & 69 & 1 & 5 & 2,72 & 1,02 \\
\hline $\begin{array}{l}\text { MOTIV14 El EEES me obliga a formarme para poder adaptarme a } \\
\text { los nuevos requerimientos de este modelo }\end{array}$ & 68 & 1 & 5 & 3,44 & 1,01 \\
\hline $\begin{array}{l}\text { MOTIV15 Para mi es un valor importante dedicar parte de mi tiempo } \\
\text { a formarme para la mejora de mi docencia }\end{array}$ & 70 & 3 & 5 & 4,34 &, 63 \\
\hline N válido (según lista) & 64 & & & \\
\hline
\end{tabular}

Para identificar las motivaciones iniciales del profesorado ante la formación en metodologías activas de enseñanza, nos centramos en las puntuaciones medias más altas. Así el valor más alto es el del ítem 9, 'me preocupa que el alumnado logre un aprendizaje y desarrollo de las competencias personales y profesionales' $(4,75)$. Al que siguen de cerca los ítems 8 , 'considero que la formación es necesaria para ser docente competente' $(4,58)$, el 5 , 'las características de mi labor docente en la universidad (trabajar con estudiantes, dar clase) me motivan por sí mismas' $(4,46)$ y el 15 , 'para mí es un valor importante dedicar parte de mi tiempo a formarme para la mejora de mi docencia' $(4,34)$. 
Los valores alcanzados por la desviación típica en estos ítems (entre 0,47 y 0,60), son los más bajos. Estas cuestiones no sólo gozan de la aceptación de los profesores, sino que, además, presentan menor variabilidad en las respuestas. No obstante el valor más bajo de la desviación típica lo ha obtenido el ítem $9(0,72)$. Tal puntuación, junto al valor de la media más alto $(4,75)$, le dotan de gran consistencia: es la cuestión que goza del mayor nivel de consenso. El profesorado manifiesta claramente su preocupación para que el alumnado logre un aprendizaje y desarrollo de las competencias profesionales. Aspecto que se confirma con el análisis cualitativo de la pregunta abierta, recogido en 26 unidades textuales. Al referirse a la participación del alumnado reflejan la preocupación por convertirle en un agente activo, consciente y responsable de su proceso educativo.

Mujer: Adquisición de una metodología que permita un aprendizaje más activo del alumno... Un aprendizaje activo permite al alumno ser más consciente de su evolución en una materia, de sus capacidades y de las posibilidades que esa materia le puede ofrecer para su futura vida profesional, además de asumir una responsabilidad más activa en dicho proceso (I. A.I S1/MOTIGU).

(M. profesora 2) Me gusta dar clases y motivar al alumnado; me encantaría promover su implicación personal en el aprendizaje, sobre todo, en la problemática social relacionada con mis disciplinas (sistema penal y penitenciario). Creo que esta metodología puede mejorar mis capacidades para avanzar hacia ese objetivo.

(1B1A profesora 3) Considero necesario mantener una formación constante y estar al día para mejorar profesionalmente como docente.

En las puntuaciones medias el valor más bajo corresponde al ítem 3, 'me he involucrado en este proceso formativo para facilitar mi promoción profesional, mejorar mi currículum, los incentivos...' $(2,51)$, seguido muy de cerca por el ítem 6, 'me motiva el status del profesor universitario (reconocimiento social...)' (2,54), el 7, 'me siento empujado/a institucionalmente a participar en este tipo de iniciativas' $(2,61)$ y el 13, 'la formación docente aumenta mi prestigio como profesor universitario' (2,72). Además su desviación típica es la más alta (entre 1,02 y 1,15). Son los ítems del cuestionario de mayor variabilidad, teniendo por consiguiente, el menor grado de consenso entre el colectivo. Tampoco se han recogido evidencias en la pregunta abierta que hagan referencia a las motivaciones extrínsecas de los docentes ante su formación. Como afirma Kenneth (2002), las recompensas extrínsecas pasan a un primer plano cuando los beneficios de los trabajadores no son suficientes, cuando surgen temas de injusticia social y cuando afrontan opciones importantes. Se diluyen en el resto del tiempo, y las recompensas intrínsecas ocupan el primer plano en el trabajo diario. En raras ocasiones el trabajo equivale a un sprint corto para lograr una recompensa. A largo plazo, las personas necesitan recompensas intrínsecas para seguir adelante y rendir al máximo. En este sentido, los ítems de la tabla responden a estas dos percepciones de la motivación: la intrínseca y la extrínseca. La motivación intrínseca está reflejada en los ítems 2, 4, 5, 8, 9, 10, 11, 15 y lleva al profesorado a buscar la formación por sí misma. Mientras que los ítems 3, 6, 7, 12, 13, 14 correspondientes a la motivación extrínseca, trasladan la idea de la formación como un trámite para conseguir recompensas, tales como, promoción profesional, incentivos etc.

El análisis categorial realizado pone de manifiesto que la categoría más sobresaliente es 'Entusiasmo Docente' (con 84 unidades textuales), que posteriormente explicaremos con el análisis factorial. Este dato refleja que la motivación intrínseca está más presente en este profesorado universitario que apuesta por el cambio metodológico. Este primer análisis descriptivo permite concluir, como muchos autores sostienen (Antón, 2005; Bastick, 2000; Biscarri et al., 2006; Guterman, 2005; Kenneth, 2002; Prince, 2006 y Sinclair, 2006), que los docentes están más motivados por componentes intrínsecos de su tarea profesional que por los de carácter extrínseco. Estos mismos estudios avalan el carácter altamente vocacional de la profesión docente en general, ya que para los profesores su principal motivación son los propios alumnos. Ejercen la docencia porque les gusta trabajar con jóvenes y ayudarles en su formación y sienten gran satisfacción viendo como con su apoyo aprenden, desarrollan su potencial y se preparan para 
actuar como adultos responsables. Por el contrario, otras investigaciones (Vezub, 2009) también defienden la existencia de docentes universitarios que eligen los cursos no por su calidad y pertinencia para su tarea, sino por la puntuación que les otorgan para el ascenso y la elección de mejores lugares de trabajo, es decir, la motivación extrínseca.

\section{Análisis factorial}

Como se mencionó anteriormente, se ha realizado un análisis factorial cuyos resultados nos permitirán profundizar en el perfil motivacional del profesorado estudiado. Técnicamente este análisis factorial se caracteriza por un coeficiente $\mathrm{KMO}$ algo bajo (0.637), aunque la significatividad es alta (,000), y por una chi-cuadrado de 188,251 y un gl de 91, en la prueba de esfericidad de Bartlett. Aunque estos datos no ofrecen excelentes condiciones para realizar un análisis dimensional, nos centramos en ver qué tipo de estructuración presenta la escala y analizar qué grupos de ítems tienden a ir juntos formando factores. En primer lugar nos fijamos en el número de factores hallados y la varianza explicada (Tabla 2). El factor 1 explica el 13,03\%; el factor 2, el 12,55 \%; y el factor 3, el 8,68 \%; de modo que los tres factores explican el 34,27\% de la varianza. Aunque no es un porcentaje muy elevado, que analizamos la estructura resultante.

Tabla 2: Varianza total explicada

\begin{tabular}{|l|r|r|r|r|r|r|}
\hline \multirow{2}{*}{ Factor } & \multicolumn{4}{|c|}{ Autovalores iniciales } & \multicolumn{3}{c|}{$\begin{array}{c}\text { Suma de las saturaciones al } \\
\text { cuadrado de la rotación }\end{array}$} \\
\hline & Total & $\begin{array}{r}\text { \% de la } \\
\text { varianza }\end{array}$ & \% acumulado & Total & $\begin{array}{r}\text { \% de la } \\
\text { varianza }\end{array}$ & \% acumulado \\
\hline 1 & 3,14 & 22,43 & 22,43 & 1,82 & 13,03 & 13,03 \\
\hline 2 & 1,92 & 13,72 & 36,16 & 1,75 & 12,55 & 25,58 \\
\hline 3 & 1,34 & 9,58 & 45,74 & 1,21 & 8,68 & 34,27 \\
\hline 4 & 1,28 & 9,19 & 54,94 & & & \\
\hline 5 & 1,17 & 8,37 & 63,31 & & & \\
\hline 6 &, 90 & 6,45 & 69,76 & & & \\
\hline 7 &, 84 & 6,01 & 75,78 & & & \\
\hline 8 &, 71 & 5,08 & 80,86 & & & \\
\hline 9 &, 63 & 4,50 & 85,37 & & & \\
\hline 10 &, 56 & 4,06 & 89,43 & & & \\
\hline 11 &, 46 & 3,33 & 92,77 & & & \\
\hline 12 &, 36 & 2,57 & 95,35 & & & \\
\hline 13 &, 34 & 2,46 & 97,81 & & & \\
\hline 14 & & 2,18 & 100,00 & & & \\
\hline
\end{tabular}

La matriz de los factores rotados ha sido realizada con el método de extracción: factorización del eje principal. Método de rotación: Normalización Varimax con Kaiser. La rotación ha convergido en 4 iteraciones. La matriz describe los ítems que constituyen cada uno de los tres factores (Tabla 3).

Los resultados permiten afirmar la existencia de tres dimensiones motivacionales en el cuestionario, aunque no se corresponden exactamente con las inicialmente proyectadas, guardan un cierto paralelismo. El primer factor podría estar relacionado con las dimensiones preestablecidas "carencias competenciales" y "desarrollo personal", creando una nueva dimensión a la que denominamos "entusiasmo docente"; el segundo factor es el más evidente, ya que encaja con "promoción profesional", y por último el "utilitarista" crea una nueva dimensión. Estas denominaciones engloban el significado del contenido de las agrupaciones de ítems; y coinciden con algunas de las categorías emergidas del análisis cualitativo de las unidades textuales que a continuación se detalla. 
Tabla 3: Matriz de factores rotados

\begin{tabular}{|l|c|c|c|}
\hline & \multicolumn{3}{|c|}{ Factor } \\
\hline & 1 & 2 & 3 \\
\hline MOTIV15 &, 69 & & \\
\hline MOTIV08 &, 63 &, 23 & \\
\hline MOTIV09 &, 54 & & \\
\hline MOTIV11 &, 49 & & \\
\hline MOTIV05 &, 44 & & \\
\hline MOTIV04 &, 24 & & \\
\hline MOTIV06 & &, 91 & \\
\hline MOTIV13 & &, 51 & \\
\hline MOTIV03 & &, 48 & \\
\hline MOTIV07 &,- 20 &, 34 & \\
\hline MOTIV10 & &, 29 & \\
\hline MOTIV14 & & &, 73 \\
\hline MOTIV12 & &, 29 &, 65 \\
\hline MOTIV02 & & & \\
\hline
\end{tabular}

\section{Análisis categorial}

Los encuestados a través de la pregunta abierta han podido expresar la reflexión en torno a sus motivaciones. En este sentido, el análisis de contenido de las respuestas se ha realizado con el programa informático Nvivo 8.0. Del análisis de unidades textuales generadas ha permitido construir unas categorías que han configurado un sistema categorial.

La denominación y descripción de cada una de ellas se ha extraído con rigor científico del análisis inductivo del contenido de las unidades textuales. La categoría más consistente tanto por el número de unidades textuales como por el contenido de la misma es 'entusiasmo docente'.

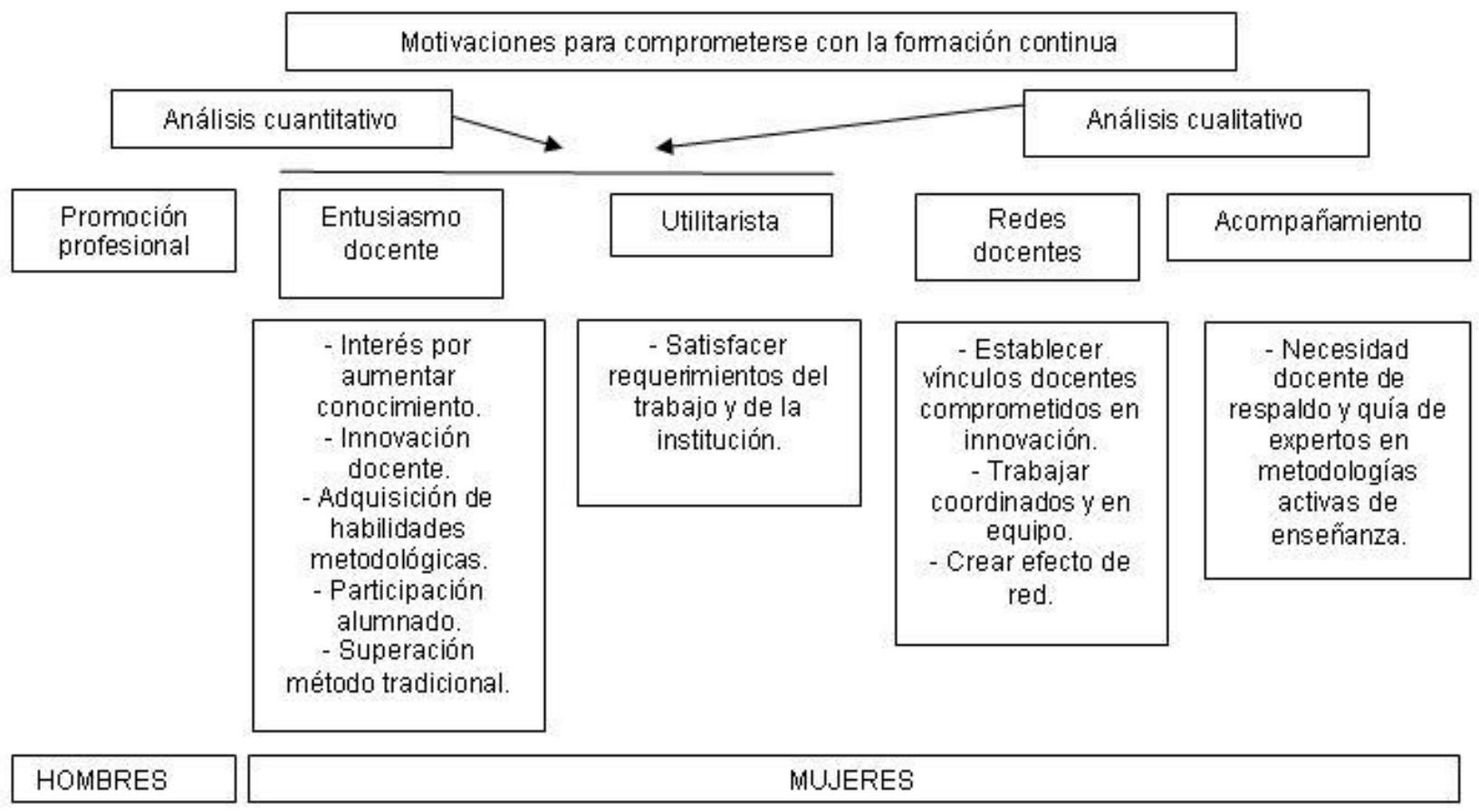

Fig. 1: Sistema categorial emergente del análisis cualitativo de contenido. 
Esta categoría en particular, contiene unas sub-categorías que explicitan más concretamente su significado. Esta diversidad permite especificar las motivaciones intrínsecas presentes en el proceso formativo. A continuación trataremos detalladamente de integrar los análisis cuantitativo y cualitativo para dar una mayor profundidad y concreción a las tres categorías definidas. El factor 1 al que hemos denominado entusiasmo docente, está formado por los ítems mostrados en la Tabla 1.

Tabla 4: Ítems que forman el factor entusiasmo docente

MOTIV05 Las características de mi labor docente en la universidad (trabajar con estudiantes, dar clase) me motivan por sí mismas

MOTIV08 Considero que la formación es necesaria para ser docente competente

MOTIV09 Me preocupa que el alumnado logre un aprendizaje y desarrollo de las competencias personales y profesionales

MOTIV11 Propuestas como ERAGIN me resultan motivadoras para mi desarrollo profesional

MOTIV15 Para mí es un valor importante dedicar parte de mi tiempo a formarme para la mejora de mi docencia

En esta categoría existe una clara unanimidad entre los docentes al explicitar el entusiasmo docente: interés por mayor conocimiento, innovación docente, adquisición de habilidades y destrezas metodológicas, implicación del alumnado y superación del método tradicional. Algunas de las unidades textuales que las evidencian (un total de 84 unidades textuales) son:

(A. M. profesora 4) Después de haber realizado unos cuantos cursos organizados por el SAE, y habiendo empezado a aplicar herramientas de innovación docente, estaba interesada a emprender este proyecto con una dimensión mayor que los cursitos de 20-30h. Pienso que tras realizar el Proyecto Eragin tendré una visión más clara de las posibilidades y las propias competencias a desarrollar por el profesorado en las aulas.

(029 profesor 5) A nivel personal estoy comprometido con las metodologías activas desde hace bastantes años y este programa considero que es una buena oportunidad para revisar los que estás haciendo y para ver nuevas ideas. Además los nuevos planes son un reto personal que implica un nuevo esfuerzo de adaptación para el que todas las ayudas son pocas.

(a-m-h profesora 6) Mejorar la impartición de los conocimientos y lograr una mayor participación y motivación en los alumnos. Pretendo lograr clases más dinámicas y participativas, en un ambiente de trabajo que facilite para los alumnos, la consecución de objetivos y el alcanzar las competencias previstas.

(A. $R$. profesora 8) La razón es que la clase expositiva entra en una rutina que hace bajar la motivación del alumnado y la mía propia.

El factor 2 llamado promoción profesional lo componen los ítems mostrados en la Tabla 2 y el factor 3 sólo agruparía dos ítems por lo que es más secundario en el plano estadístico y lo reconocemos bajo la etiqueta utilitarista: Como hemos explicado anteriormente no disponemos de unidades textuales que reflejen esta dimensión extraída del análisis factorial.

Por el contrario, en el análisis cualitativo esta tercera dimensión goza de mayor protagonismo entre los docentes (11 unidades textuales). Varios docentes han visto útil este proceso de formación de cara a la estructuración de las nuevas titulaciones que implementa el proceso Bolonia. De alguna manera, más de uno se ha sentido presionado y se ha acercado a este proceso para satisfacer estos requerimientos o exigencias provenientes de la institución universitaria como también de su propio trabajo como docente. 
Tabla 5: Ítems que forman el factor promoción profesional

MOTIV03 Me he involucrado en este proceso formativo para facilitar mi promoción profesional, mejorar mi currículum, los incentivos...

MOTIV06 Me motiva el status del profesor universitario (reconocimiento social...)

MOTIV07 Me siento empujado/a institucionalmente a participar en este tipo de iniciativas

MOTIV10 Me interesa dinamizar grupos de docentes preocupados por el cambio metodológico

MOTIV13 La formación docente aumenta mi prestigio como profesor universitario

Tabla 6: Ítems que forman el factor utilitarista

MOTIV12 Participo en esta formación para no quedarme al margen de los cambios

MOTIV14 EI EEES me obliga a formarme para poder adaptarme a los nuevos requerimientos de este modelo

(D. profesor 9) La inmediatez de implementación del nuevo espacio europeo exige cambios (si se quiere hacer bien) por lo que deberemos cambiar nuestra forma de hacer las cosas.

(JGR profesor 10) Próxima implementación del GRADO conforme a las exigencias del EEESestilo Bolonia.

Respecto al ítem no 4, 'mi preocupación principal es desarrollarme como persona (crecimiento, compromiso, responsabilidad personal...)', y el $\mathrm{n}^{\circ} 2$, 'me he apuntado a este proceso para cubrir mis carencias competenciales (falta recursos pedagógicos, habilidades docentes...)', nos generan ciertas dudas por sus valores estadísticos. Hemos considerado oportuno no ubicarlos por el momento en ninguna de las dimensiones existentes. Los tendremos en cuenta en una fase posterior de la investigación que estamos realizando, por ejemplo integrándolos en una entrevista.

Resumiendo, el factor $n^{0} 1$ - entusiasmo docente - y el factor $n^{0} 3$ - utilitarista - coinciden con dos de las categorías provenientes del análisis cualitativo, denominadas de la misma manera. Aun así, mediante el análisis cuantitativo solo se han detectado 2 de las 4 dimensiones obtenidas a través del análisis de contenido. Las dos categorías complementarias que representan otros dos tipos de motivaciones iniciales del profesorado son: 'Redes docentes' y 'Acompañamiento'. Alguno de los docentes que han participado en el programa ERAGIN han mostrado su interés por crear de redes docentes para trabajar de manera coordinada y en equipo (En total hemos reunido once unidades textuales).

(20001229 profesora 12) Zaila da horrelako metodologia bat aplikatzea norbanako gisa eta horregatik oso interesgarria ikusten dut eta baita lagungarria ere Unibertsitateak martxan jarri duen ekimena. Talde bat sortzeak ere animatzen nau, horrela ez garelako bakarrik sentituko eta kideen esperientziak ere guretzat baliagarriak izango direlako. Ikastegi eta Sailen aurrean ere gure lanak babes handiagoa izango du eta talde sendo baten bermea, ezta?

(I. L. profesora 13) Oso inportantea izan da, kurtsoa eta programa eskatzeko momentuan, irakaskuntza aktiboaren aldeko lan-taldearen partaidea izateko nahia.

En segundo lugar, existen docentes que se han sentido motivados por verse respaldados por profesionales expertos en metodologías activas de enseñanza durante este proceso de cambio metodológico. Valoran este punto como una singularidad de este programa frente a otros (En total hemos categorizado 4 unidades textuales):

(BBAA profesor 14) A parte de los contenidos del curso lo que más me motiva es el hecho de tener un tutor con el que poder compartir, revisar y corregir el trabajo. Hasta ahora en todos los 
cursos, la aplicación era sin seguimiento y considero que el esfuerzo de aprender, también en el profesorado exige de supervisión y corrección en su caso así como el de la valoración final. ¿Qué aprovechamiento de los cursos hacemos el profesorado? ¿Cuánto de ese aprendizaje llega a las aulas? y ¿en qué medida mejora la enseñanza?

\section{Comparación de medias}

Teniendo en cuenta los datos de identificación de la muestra requeridos y sin perder de referente los objetivos establecidos en este estudio, examinamos cómo se comportan estas 3 dimensiones obtenidas en el análisis factorial tiendo en cuenta las variables independientes 'sexo', 'antigüedad' y 'área de conocimiento ejercen docencia'. (Véanse tablas 7, 8 y 9). A partir de las medias, podemos comprobar que las mujeres puntúan más alto en entusiasmo docente que los varones, aunque las diferencias no son muy grandes. En cambio, los varones obtienen mayor puntuación en comparación con las mujeres en promoción profesional. Nuestro resultado coincide con el estudio de Bruisman y Jansen (2010) donde se evidencia que el colectivo docente femenino muestra significativamente mayor motivación intrínseca que el masculino. Y con el de Bastick (2000) que comparando la motivación de docentes universitarios masculinos y femeninos en relación al género y la cultura, sostiene que las mujeres profesoras se mueven por motivaciones intrínsecas mientras que los hombres se decantan más a favor de las extrínsecas. En la misma línea, Prince (2006) apunta que las mujeres docentes gozan de mayor satisfacción laboral.

Las puntuaciones notoriamente más altas en entusiasmo docente ponen de manifiesto que la motivación hacia la práctica educativa es constante. De todas formas los resultados no son significativos. En todos los intervalos de edad los valores máximos se han aglutinado alrededor de 4,4. Sin embargo, según la investigación comparativa realizada por Bastick (2000), los profesores más adultos apuntaban más alto en motivación intrínseca: son profesores con más experiencia y este tipo de motivación anima a seguir en la profesión. Como consecuencia, y tal como nuestros datos indican, la motivación se incrementa con la edad.

Tabla 7: Relación variable sexo

\begin{tabular}{|c|c|c|c|c|c|c|c|c|c|}
\hline Sexo & \multicolumn{3}{|c|}{ Promoción profesional } & \multicolumn{4}{c|}{ Utilitarista } & \multicolumn{3}{c|}{ Entusiasmo docente } \\
\hline & $\mathrm{N}$ & Media & D.T. & $\mathrm{N}$ & Media & D.T & $\mathrm{N}$ & Media & D.T. \\
\hline Mujer & 46 & 2,83 &, 63 & 44 & 3,51 &, 86 & 46 & 4,53 &, 38 \\
\hline Hombre & 25 & 3,00 &, 73 & 22 & 3,31 &, 77 & 25 & 4,32 & 4,46 \\
\hline Total & 71 & 2,89 &, 67 & 66 & 3,44 &, 83 & 71 & 4,46 &, 42 \\
\hline
\end{tabular}

Tabla 8: Relaciones según la antigüedad docente

\begin{tabular}{|c|c|c|c|c|c|c|c|c|c|}
\hline Antigüedad docente & \multicolumn{3}{|c|}{ Promoción profesional } & \multicolumn{3}{|c|}{ Utilitarista } & \multicolumn{3}{c|}{ Entusiasmo docente } \\
\hline & Z & B.B. & D.T. & Z & B.B. & D.T & Z & B.B. & D.T. \\
\hline $2-7$ & 7 & 2,97 &, 58 & 7 & 3,07 & 1,01 & 7 & 4,42 &, 49 \\
\hline $8-14$ & 16 & 2,92 &, 68 & 14 & 3,50 &, 67 & 16 & 4,47 &, 38 \\
\hline $15-24$ & 26 & 2,86 &, 61 & 24 & 3,47 &, 92 & 26 & 4,48 &, 41 \\
\hline $25-33$ & 18 & 2,76 &, 63 & 17 & 3,58 &, 83 & 18 & 4,47 &, 44 \\
\hline Total & 67 & 2,86 &, 62 & 62 & 3,46 &, 85 & 67 & 4,47 &, 41 \\
\hline
\end{tabular}

Tabla 9: Relación variable área de conocimiento.

\begin{tabular}{|l|c|c|c|c|c|c|c|c|c|}
\hline \multicolumn{1}{|c|}{ Área de conocimiento } & \multicolumn{3}{|c|}{ Promoción profesional } & \multicolumn{3}{c|}{ Utilitarista } & \multicolumn{3}{c|}{ Entusiasmo docente } \\
\hline & $\mathrm{N}$ & Media & $\mathrm{D} . \mathrm{T}$. & $\mathrm{N}$ & Media & D.T & $\mathrm{N}$ & Media & D.T. \\
\hline Ciencias Sociales & 40 & 3,01 &, 71 & 37 & 3,40 &, 77 & 40 & 4,38 &, 43 \\
\hline Ciencias Humanas & 5 & 2,28 &, 41 & 4 & 2,87 & 1,18 & 5 & 4,68 &, 52 \\
\hline Ciencias Experimentales & 10 & 2,46 &, 59 & 10 & 3,35 & 1,02 & 10 & 4,42 &, 44 \\
\hline Ciencias Técnicas & 10 & 3,16 &, 54 & 10 & 4,00 &, 47 & 10 & 4,72 &, 16 \\
\hline Ciencias de la Salud & 6 & 2,90 &, 27 & 5 & 3,30 &, 97 & 6 & 4,50 &, 37 \\
\hline Total & 71 & 2,89 &, 67 & 66 & 3,44 &, 83 & 71 & 4,46 &, 42 \\
\hline
\end{tabular}


En referencia a las motivaciones extrínsecas, las diferencias son muy pequeñas, pero cabe resaltar que las puntuaciones más altas las han otorgado los docentes de 2 a 7 años de experiencia $(2,97)$. Puede que este dato refleje una cierta inestabilidad laboral. Las puntuaciones más altas en las cinco áreas las ha obtenido el entusiasmo docente, pero existiendo diferencias entre ellas: Ciencias Técnicas ha obtenido la mayor puntuación (4.7 sobre 5) frente a Ciencias Sociales que ha obtenido la menos alta (4.38). Este resultado confirma el peso mayor obtenido por esta dimensión en el análisis tanto factorial como categorial.

En relación a promoción profesional, el área de conocimiento de Ciencias Técnicas ha sido también la que mayor valor ha obtenido $(3,16)$, mientras que el valor mínimo está en Ciencias Experimentales $(2,46)$. Por último, en la tercera variable utilitarista se repite la situación anterior, el valor máximo se lo llevan las Ciencias Técnicas, y el mínimo se queda en Ciencias Experimentales. Podemos afirmar que en el programa ERAGIN, impulsor del cambio metodológico en la enseñanza y aprendizaje, el grupo de profesores que lo lidera pertenece al área de las Ciencias Técnicas, y no al de Ciencias Sociales como cabría esperar. Los primeros han obtenido las puntuaciones más altas en entusiasmo docente, en utilitarista y también en la dimensión de promoción profesional.

\section{CONCLUSIONES}

Del estudio llevado a cabo se obtiene algunas de las conclusiones, de las cuales las más sobresalientes se detallan en lo que sigue.

1.- En el momento de dar comienzo un proceso de formación basado en el cambio metodológico, los docentes declaran tener más de un tipo de motivación. Las razones para comprometerse con el proceso ERAGIN son varias: sentir entusiasmo docente, promocionarse profesionalmente, razones utilitaristas, crear redes docentes y sentirse acompañados.

2.- Sin embargo, una que cobra mayor protagonismo, entusiasmo docente. Los docentes tienen como objetivo principal incrementar su competencia en torno a las metodologías activas de enseñanza. El cambio de pensamiento vivenciado en el núcleo de la comprensión y la praxis educativa, creen que incrementará el compromiso y auto-aprendizaje de sus alumnos. Por ello, haciendo suyas estas habilidades creen poder lograr una mejora e innovación en la enseñanza. Al mismo tiempo han valorado muy satisfactoriamente la ayuda de los expertos en esta innovación educativa como una singularidad de este programa ERAGIN. Esta conclusión coincide con la obtenida por Bruinsma y Jansen (2010): el entusiasmo docente está positivamente relacionado con la calidad del programa de formación y las experiencias docentes satisfactorias en el aula.

3.- En relación al sexo, tanto las mujeres como los hombres participantes han demostrado una alta motivación intrínseca. No obstante, tal y como lo demuestran los análisis factoriales y categoriales, a pesar de que la diferencia no es muy grande, las mujeres docentes muestran un mayor entusiasmo docente frente a los hombres. Estos en cambio, dan más importancia a las motivaciones extrínsecas que las mujeres, como por ejemplo, a la promoción profesional.

4.- Por lo que respecta a la antigüedad docente, debemos decir que los resultados no son significativos. La verdad es que entusiasmo docente ha sobresalido frente a las otras motivaciones en todos los intervalos de edad establecidos. Esto demuestra la motivación intrínseca y constante del grupo de docentes ERAGIN hacia la profesión. Las motivaciones extrínsecas en general no gozan de valores muy altos; aunque si bien son diferencias mínimas, la puntuación más alta en la categoría promoción profesional se adscribe entre los profesores noveles. Este dato puede deberse a que enfocan la formación como un trámite, ya que el reconocimiento por ello puede ayudarles a mejorar su situación administrativa-profesional dentro de la universidad.

5.- En las diversas áreas de conocimiento se han registrado valores parecidos en las tres dimensiones extraídas del análisis cuantitativo: la categoría que ha tenido mayor consenso ha sido entusiasmo docente, mientras que utilitarista es la que ha mostrado más variabilidad. 


\section{REFERENCIAS}

Añez, S. Cultura organizacional y motivación laboral de los docentes universitarios. Centro de Investigación de Ciencias Administrativas y Gerenciales: 1, 110-126 (2006).

Antón Ares, P. Motivación del profesorado universitario para la aplicación de las propuestas metodológicas derivadas de la utilización de las tecnologías de la información y de la comunicación en la docencia. Rev. Latinoam. de Tecnol. Educativa: 4 (1), 101-110 (2005).

Barberá, E. y Mateos, P.M. Investigación sobre psicología de la motivación en las universidades españolas. REME: 3(5-6), 2-6 (2000).

Bastick, T. The measurement of Teacher Motivation: Cross-Cultural and Gender Comparisons, 29th Annual Meeting of the Society for Cross-Cultural Research: New Orleans, USA (2000).

Biscarri Gassió, J. Motivaciones de los profesores respecto a su formación permanente. Revista Interuniversitaria de Formación del Profesorado: 18, 221-237 (1993).

Biscarri Gassió, J.; Filella, Giui, G. y Jové Monclús, G. Factores relacionados con la percepción de la calidad docente del profesorado universitario. Revista Interuniversitaria de Formación del Profesorado: 20 (3), 287-309 (2006).

Bruinsma, M. y Jansen, E. Is the motivation to become a teacher related to pre-service teachers' intentions to remain in the profession? European J. of Teacher Education: 33 (2), 185-200 (2010).

De la Fuente Arias, J. Perspectivas recientes en el estudio de la motivación: la Teoría de la Orientación de Meta. Revista electrónica de Investigación Psicoeducativa: 2(1), 35-62 (2004).

González Fernández, A. Modelos de motivación académica: una visión panorámica. REME: 10 (25), 226 (2007).

González Torres, $\mathrm{M}^{\mathrm{a}} \mathrm{C}$. Claves para favorecer la motivación de los profesores ante los retos educativos actuales. Estudios sobre educación: 5, 61-83 (2003).

Kenneth, W. Th. La motivación intrínseca en el trabajo. Editorial Universitaria Manuel Areces, Madrid, España (2002).

Lozano, S. y Repetto, E. Motivación y desarrollo profesional. REME: 10(25), 62-86 (2007)

Manuel, J. y Hughes, J. 'It always been my dream': exploring pre-service teachers' motivations for choosing to teach. Teacher Development: 10 (1), 5-24 (2006).

Muchinsy, P. Psicología Aplicada al Trabajo. Editorial Thompson, Madrid, España (2000).

Ollarves, Y. Cultura organizacional y propiedades motivantes del puesto de trabajo en una institución de educación superior. Investigación y Postgrado: 21 (1), 125-151 (2006).

Rodríguez Fernández, A.; Zarco Martín, V. y González González, J. Psicología del trabajo, Pirámide, Madrid, España (2009).

Rosales López, C. En torno a la Formación Permanente del profesor: Motivos y procesos. Comunicación y Pedagogía: 212, 26-29 (2006).

Ryan, J. Continuous professional development along the contininuum of lifelong learning, Nurse Education Today: 23, 498-508 (2003).

Silvero,M. Motivación y calidad docente en la universidad. EUNSA, Pamplona, España (2006).

Sinclair, C.; Dowson, M. y Thistleton-Martin, J. Motivations and profiles of cooperating teachers: Who volunters and why? Teaching and Teacher Education: 22, 263-279 (2006).

Uwamariya, A. y Mukamurera, J. Le concept de développement professionnel en enseignement: approches théoriques. Revue des sciences de l'education: 31(1), 133-155 (2005).

Vezub, L. F. Notas para pensar una genealogía de la formación permanente del profesorado en la Argentina. Revista Mexicana de Investigación Educativa: 14 (42), 911-937 (2009). 